\title{
La atención al cuidador. Una visión interdisciplinaria
}

\author{
Esther Secanilla, María Bonjoch, Margarida Galindo y Laura Gros \\ Residència i Centre de Dia d'Horta, Barcelona (España)
}

\begin{abstract}
En una aproximación global a la atención de los cuidadores de personas mayores con demencia que viven en el domicilio, es fundamental promocionar su bienestar personal, social y emocional, así como proporcionar herramientas que mejoren la calidad de vida del cuidador. Con este objetivo se presenta la experiencia del CDD y Residencia de Horta, Barcelona. Para la definición de la muestra se realizó una revisión documental del archivo en el periodo comprendido entre 2004 y 2010. Para el análisis de las entrevistas semiestructuradas de ingreso, seguimiento y asesoramiento, se realizó una recogida de información a través del diario de registros. Otras estrategias aplicadas fueron cuestionarios de satisfacción, a partir de las necesidades detectadas por parte de los cuidadores que asistieron al Grupo de Ayuda Mutua en el período indicado, así como el cuestionario validado de sobrecarga del cuidador (Zarit y Zarit, 1982). Asimismo, se realizó una sistemática observación participante del grupo focal del GAM. Las entrevistas se administraron a cuidadores principales que asistían al GAM. Los cuestionarios de satisfacción y los test fueron cumplimentados por la muestra. Se demuestra que a partir de las acciones de prevención y seguimiento así como la formación psicoeducativa dirigida a cuidadores se favorece la permanencia de los usuarios en el propio domicilio, se mejora la calidad de vida de éstos y de sus cuidadores.
\end{abstract}

Palabras clave: Cuidadores, GAM, sobrecarga del cuidador, calidad de vida, Residencia y CDD Horta.

The attention of caregiver. An intersdisciplinary vision. In a global approach to the attention of carers of older people with dementia living at the elder's home, it is essential to promote their personal, social and emotional wellbeing as well as provide them with tools that improve their quality of life. With this goal we present the experience of CDD and Residencia de Horta, Barcelona. A documentary review of the file was made, covering the period between 2004 and 2010 to define the sample. For the analysis of the semistructured admission interviews, monitoring and mentoring, a research was made through the daily records. Other strategies used were fulfilment questionnaires as well as the validated questionnaire of caregiver's burden (Zarit and Zarit, 1982). Likewise, there was a systematic active observation of the GAM group. The interviews were made to the caregivers who attended the GAM. It is proven that actions of prevention and monitoring as well as psycho-educational training designed for carers promotes the user's staying at their home, improves their quality of life and their caregiver's.

Key words: Caregivers, GAM, caregiver burden, quality of life, Residence and CDD Horta.

Correspondencia: Esther Secanilla. Departamento de Psicología. Residencia y CDD Horta, Josep Sangenís, 75. 08032 Barcelona (España). E-mail: Esther.Secanilla@uab.cat 
En España actualmente hay cerca de 600.000 enfermos de Alzheimer, siendo la primera entre las enfermedades neurodegenerativas. Es una demencia que aparece en el quinto o sexto decenio de la vida progresando cada vez más con gran rapidez comenzando con síntomas neurológicos hasta llegar a la dependencia total.

El cuidador es la persona encargada del cuidado de estos enfermos, dedicando en su mayoría las $24 \mathrm{~h}$ a él. Poco a poco va descuidando sus propias necesidades para ocuparse por completo de éste. Por esto, es imprescindible diseñar e implementar programas institucionales para la detección e intervención de la sobrecarga psicofísica en los cuidadores de enfermos de Alzheimer.

El cuidador se hace cargo a menudo de aspectos económicos, sociales y mentales a medida que la enfermedad evoluciona y el nivel de dependencia aumenta (Leturia y Yanguas, 1999). Gracias a la contribución del cuidador informal se favorece la integración en su entorno, potenciándose las capacidades aún conservadas y supliendo las pérdidas, consiguiendo mantener por más tiempo y en mejores condiciones los mayores en su propia comunidad (Artaso, Goñi y Gómez, 2001a; Pérez, 1997).

La Confederación Española de Asociaciones de Familiares de Alzheimer, en el 2003, distingue tres etapas en el cuidado del mayor: Preparación-adquisición -se desarrolla de forma progresiva, en que el cuidador apenas es consciente de que está asumiendo el rol de cuidador (Crespo y López, 2007) -; promulgación-actuación -el cuidador asume y ejerce su rol como cuidador haciendo frente a las necesidades propias del cuidado pudiendo darse o no la institucionalización del mayor -; abandono del rol muerte del paciente teniendo, el cuidador, que readaptarse a vivir sin éste -.

Con la experiencia que presentamos, los cuidadores aportan causas diversas de enfermedad a las que deben atender dejando de lado muchas veces sus propias necesidades, obligaciones, intereses, y apartándose poco a poco de las redes de relaciones sociales. En este sentido, se especifican diversas situaciones en el filme cuidadores (Cuidadores 2011) La realidad es que los recursos sociales para atender las necesidades de las personas mayores son muy escasos, y sin estos cuidadores, llamados informales, la red de ayuda psicosocial se reduciría al mínimo. Pero estos cuidadores necesitan también cuidados, pues se enfrentan, diariamente, a un número elevado de situaciones difíciles, exigencias, demandas por parte del enfermo, situaciones que conllevan implicaciones negativas tanto a nivel físico como emocional. Por este motivo, desde la residencia i CDD Horta, hemos querido ofrecer recursos, estrategias, y sesiones psicoeducativas para potenciar las habilidades del propio cuidador para con su persona cuidada, además de concienciarse de su autocuidado, su propia autonomía y su seguridad en el domicilio. Este trabajo se lleva a cabo desde una perspectiva interdisciplinaria.

El trabajo interdisciplinario hace referencia a «aquella implicación colectiva de un conjunto de profesionales diferenciados en el tipo de información y experiencia 
que aportan y en el tipo de intervención que realizan pero que están enfrentados a un objeto común y que están igualados en el momento de tejer sus aportaciones» (Casals, De Vicente, Garriga y Tabueña, 2005:71).

Las personas cuidadoras de la muestra muchas veces olvidan sus propias necesidades, tan interesados como están en cuidar a los otros. Por este motivo se han desarrollado diversos tipos de programas -entre los que se encuentran los programas psicoeducativos- que les sirven de apoyo. Diversos autores describen la tipología y beneficios sobre dichas medidas de prevención (Diputació, 2004; Crespo y López, 2007). Tipologías de programas hay diversas: Programas de apoyo formal (descarga en residencias, estancias en residencias, servicios de ayuda en el domicilio), Programas psicosocioeducativos (ofrecen información y formación sobre la enfermedad, los problemas asociados a ella, la progresión, tipología), Grupos de Ayuda Mutua (grupos de soporte -y algunos educativos-, que se organizan a partir de encuentros de personas que están en la misma situación y pretenden crear una red de apoyo. Son coordinadas por profesionales y la periodicidad suele determinarse previamente. Estos grupos debieran dirigirse por profesionales puesto que en caso contrario podrían derivarse efectos negativos sobre el propio cuidador o sobre otros cuidadores al compartir emociones negativas (Crespo y López, 2007:86).

En el Centro donde presentamos la experiencia, además del GAM, ofrecemos otras alternativas de acompañamiento a los familiares, realizando el seguimiento de su proceso personal a través de terapia individual. En este sentido, y combinando esta estrategia con la anterior, ofrecemos nuestra experiencia.

Partimos de que la calidad de vida del usuario está en dependencia de la calidad de vida que pueda ofrecerle su cuidador principal. Para ello deben diseñarse estrategias de atención a los cuidadores de personas con demencias que viven en el domicilio, proporcionándoles bienestar emocional, personal y social que mejoren su propia atención, estrategias que al mismo tiempo mejoran la calidad de vida del cuidador y de la persona cuidada, y en consecuencia la calidad de atención del servicio desde las cuales se ofrecen.

El cambio demográfico en estos últimos años, el aumento de la esperanza de vida ha provocado un incremento significativo del peso social de las personas mayores en nuestra sociedad. Este incremento ha venido acompañado de una mayor diversidad del colectivo de personas mayores. De aquí la introducción de un nuevo discurso: el de las "personas mayores activas".

En nuestro entorno, y con el objetivo de atender esta diversidad, la Generalitat a través del Departamento de Benestar Social i Familia, responde con un amplio abanico de necesidades a través del Sistema de Servicios Sociales que ha dado un paso adelante con la aprobación en octubre del 2007 de la nueva ley de Servicios Sociales, el impulso a 
iniciativas que promueven la participación de la gente mayor. La creación de la oficina de la Gent Gran activa i el Consell de la Gent Gran a Catalunya.

Han sido dos leyes las más destacadas en estos últimos tres años que han afectado al ámbito de actuación de los Servicios Sociales. La gran ventaja es que por primera vez tenemos todos los recursos bien ordenados, en una cartera de servicios, la lástima es la falta de presupuesto que han aprobado para ponerla en marcha.

Por un lado, se rige por el principio de universalidad en el acceso a los Servicios, hecho que supone la obertura del sistema a las clases medias, que hasta ahora quedaban excluidas por razones de renta. Por el otro lado, la nueva ley que reconoce el derecho Subjetivo Servicios Sociales, roto con la situación que hasta ahora había caracterizado el sector. La normativa anterior establecía el derecho a los Servicios Sociales pero quedaba supeditado a la disponibilidad de recursos económicos y no a la necesidad de cada persona.

Otra Ley que ha cambiado el panorama social y de salud, La Ley de la Dependencia 14/2/2006, entra en vigor 1/1/2007.Norma estatal, el despliegue es competencia de las comunidades autónomas.

En esta investigación, nos centramos en algunas experiencias llevadas a cabo en la Residencia Horta. Este Centro, junto con el Centro de Día -inaugurada el año 1992, años de las Olimpiadas de Barcelona-, presta servicio a las personas mayores adaptadas a las necesidades asistenciales de los usuarios. El CDD tiene capacidad de 30 plazas con domicilio C/Josep Sangenís, 75, Barcelona. La Residencia Asistida d'Horta es un servicio de acogimiento residencial con carácter permanente o temporal de asistencia integral a las actividades de la vida diaria para personas mayores con dependencia. Tiene capacidad de 70 plazas.

\section{METODO}

En el diseño de la investigación, y partiendo de los objetivos que se especifican en el siguiente epígrafe, se decidieron los instrumentos a utilizar -registro documental, entrevistas, observación del grupo focal y cuestionarios validados-, se procedió a seleccionar la muestra, la recogida de los datos y por último el análisis de éstos, partiendo de una reflexión constante y de la interpretación de los resultados, combinando metodología cuantitativa y cualitativa.

Nuestro foco es la atención a las personas cuidadoras de enfermos, desde una visión interdisciplinaria, considerando la necesidad de ofrecer atención de calidad para mejorar su bienestar emocional y físico y por lo tanto la calidad de atención que recibe el enfermo.

El objetivo general de la investigación es analizar las acciones dirigidas a los cuidadores de personas con enfermedad de Alzheimer y otras en el CDD i Residencia 
Horta. A partir de este objetivo, se plantearon los siguientes objetivos específicos: Analizar el nivel de sobrecarga emocional de los cuidadores; Proponer mejoras del programa GAM del Centro a partir del análisis de los resultados obtenidos; Proporcionar mejoras en la vida diaria del cuidador y mejorar su calidad de vida.

\section{Participantes}

La muestra escogida han sido los cuidadores que atienden a sus familiares institucionalizados en el Centro de Día -algunos de los cuales han accedido a la Residencia Horta de Barcelona- y que han participado en diversas acciones ofrecidas por el equipo del profesionales técnicos del centro, o bien que han asistido al grupo de ayuda mutua (GAM), de forma periódica.

La totalidad de la muestra es de 30 de sujetos, de los cuales la mayoría permanecieron de una forma bastante regular en el GAM durante el período 2005-10 y además recibieron otros soportes de ayuda, mediante entrevistas de ingreso, seguimiento y asesoramiento.

\section{Instrumentos}

Se partió de la revisión documental del archivo del centro, para recoger datos respecto al perfil de cuidador, a los datos sociodemográficos, y otros datos de interés para el estudio. Se obtuvieron también datos a partir del registro de las actividades de los profesionales, así como de las memorias anuales del centro.

Se analizaron las entrevistas de ingreso, seguimiento y asesoramiento recogiendo datos complementarios sobre el perfil de los cuidadores, tipo de actividades que realizaban, acciones preventivas a realizar, etc. En las entrevistas de ingreso a los familiares, se les invita a asistir al GAM.

Además de una observación participante sistemática sobre el grupo focal del GAM, se diseñaron cuestionarios de satisfacción que se fueron distribuyendo a los componentes del grupo al finalizar cada período de sesiones anuales, a través de los que se extrajeron propuestas de mejora para siguientes programas, aportadas por los participantes. Además, el análisis de los datos permitió reflexionar sobre diversas necesidades de los componentes del grupo, y sobre la propia práctica profesional. Pensamos que ésta es una herramienta necesaria tanto para la reflexión como para la mejora profesional.

Se administró el cuestionario de sobrecarga del cuidador validado (Zarit y Zarit, 1982), analizando y reflexionando sobre las necesidades susceptibles de atender del grupo participante en la muestra. Este cuestionario, consta de 14 ítems que se puntúan en una escala Likert de 5 opciones de respuesta. 


\section{RESULTADOS}

A continuación exponemos el análisis de algunos de los resultados relevantes del estudio, así como las reflexiones derivadas de éste.

\section{Perfil sociodemográfico de los cuidadores participantes en el estudio}

Para poder conocer las características principales que influyen en el cuidado de los familiares enfermos, se analizó el perfil sociodemográfico de los cuidadores del estudio.

Cabe destacar que mayoritariamente acuden mujeres cónyuges del enfermo al que proporcionan el cuidado. La edad de las personas que integran la muestra oscila entre 40 y 80 años. Los recursos económicos de los familiares de estas personas suelen ser medios aunque existe una escasa ayuda por parte de los organismos públicos. Obtenemos cifras significativas, al observar que la mayor parte del día se dedican al cuidado del enfermo que se prolonga a lo largo de los años.

Entrevistas individualizadas de ingreso, seguimiento y asesoramiento con el cuidador

En nuestro centro, partimos del trabajo interdisciplinar compuesto por técnicos de diversas disciplinas, la medicina, la enfermería, el trabajo social, la psicología, la terapia ocupacional y la fisioterapia. Para llevar a cabo este trabajo interdisciplinario, mantenemos reuniones semanales para compartir, discutir, elaborar PIAI, debatir estrategias y llegar a acuerdos consensuados por el equipo, que facilitan el trabajo diario. Además, como mejora, hemos incorporado a las familias en las reuniones de PIAI. Desde las disciplinas de la psicología, la terapia ocupacional, el trabajo social y la enfermería son desde las que hemos planteado el estudio.

El objetivo del Trabajador/a Social es el de ayudar a conseguir la máxima salud social del usuario, entendiendo como a tal la situación óptima en relación a cada una de las dimensiones que configuran en entorno social de la persona. Esta profesional aportará la visión social del usuario y su familia e intervendrá individualmente con seguimientos sociales, tramitaciones de recursos y asesoramiento de la red social y sanitaria.

La psicóloga del centro realiza el diagnóstico, seguimiento y asesoramiento de los residentes y usuarios del centro, así como de sus familias. Facilita el ingreso al centro del sistema familiar y acompaña a los usuarios durante su estancia. Realiza soporte psicoemocional y cognitivo, y ofrece tanto a los residentes como a las familias estrategias para la resolución de los conflictos, espacios terapéuticos, y soporte a los casos terminales. 
Según la Asociación Castellano-Manchega de terapeutas ocupacionales la terapia ocupacional "es una profesión sociosanitaria ligada al campo de la rehabilitación e integración y se centra en prevenir, mantener y/o disminuir las consecuencias de los trastornos físicos, psíquicos, sensoriales o sociales; capacitando a la persona, con un adecuado tratamiento para alcanzar el mayor grado de independencia posible en su vida diaria, contribuyendo a la recuperación de su enfermedad y/o facilitando la adaptación a la a su discapacidad".

La Enfermería es la ciencia del cuidado de la salud del ser humano. Se dedica al diagnóstico y tratamiento de los problemas de salud reales o potenciales. El singular enfoque enfermero se centra en el estudio de la respuesta del individuo o del grupo a un problema de salud real o potencial y, desde otra perspectiva, como complemento o suplencia de la necesidad de todo ser humano de cuidarse a sí mismo desde los puntos de vista biopsicosocial y holístico.

En la recogida de datos podemos enumerar diferentes estrategias de intervención, entre ellas las entrevistas de ingreso, seguimiento y asesoramiento. Debido a las distintas situaciones en las que se va encontrando el cuidador a medida que la enfermedad avanza aparecen una serie de demandas o dudas que es necesario tratar junto al cuidador. En nuestro caso, tras las entrevistas el cuidador obtiene apoyo, información y un espacio para que la persona puede expresar sus vivencias, emociones e intentar encontrar la manera más adecuada para sobrellevar todo este proceso.

En las entrevistas de seguimiento y asesoramiento por parte de terapia ocupacional (TO) se pretende conocer la dinámica o funcionamiento general del enfermo en casa: la ejecución de las actividades de la vida diaria (AVD), conocer su historial ocupacional, sus intereses pasados y presentes, la accesibilidad en el entorno y una recogida de las demandas concretas del cuidador. Éstas son: adaptaciones en el domicilio del cuidador (baño, dormitorio, cocina), modificación de pautas de actuación en las AVD y asesoramiento en ayudas técnicas. Desde la terapia ocupacional se pretende ofrecer estrategias y recursos que potencien la autonomía del enfermo el máximo tiempo posible y que ayuden al cuidador a adaptarse mejor a la enfermedad de éste así como a posibilitar una mejor calidad de vida para ambos.

En las entrevistas de seguimiento psicológico a los familiares se destaca que los cuidadores referentes de las personas que asisten al centro de día en residencia, necesitan tiempo de adaptación a la nueva situación durante el primer periodo de ingreso en el centro. Más tarde precisan soporte para enfrentarse a las diferentes fases por las que el enfermo pasa. La comprensión de la enfermedad, de las posibles reacciones del enfermo, de lo que implica sus cuidados, se aborda desde la prevención y la anticipación de situaciones y de dotar de recursos y respuestas adecuadas al cuidador. Además, en las sesiones de seguimiento desde el ámbito psicológico se abordan cuestiones referidas con los propios recursos y competencias del cuidador, y de la importancia de cuidarse a sí 
mismo. Al mismo tiempo, es fundamental el acompañamiento en el proceso de ingreso en residencia o en los momentos de realizar descargas puntuales en otros centros. En definitiva, el abordaje de los constantes cambios y el enfrentamiento a una enfermedad que implica una degeneración de las propias funciones, y que conlleva consigo el agotamiento por parte del cuidador, la evidente fragilidad de ambos, la demanda de milagros futuros, y la evidencia de la realidad, acompañada en la mayoría de casos de un amor profundo que va aflorando durante el proceso.

Las mejoras obtenidas en cuanto al cuidador a través de las entrevistas de ingreso, seguimiento y asesoramiento son:

Mejor comprensión y consciencia de la enfermedad con la que conviven.

Mejora de la comunicación y relación entre cuidador y enfermo.

Mejor adaptación al nivel funcional del enfermo.

Mayor seguridad y accesibilidad del entorno (adaptaciones en el domicilio y ayudas técnicas).

Resolución y tramitación de recursos y mayor conocimiento de la red social y sanitaria. domicilio.

Mayor soporte psicoemocional y estrategias de resolución de conflictos en el

Como resultado de este procedimiento podemos subrayar la importancia del trabajo en equipo para poder definir estrategias de una forma coordinada y conjunta por parte de todos los profesionales implicados así como también por parte del cuidador.

\section{El GAM (grupo de ayuda mutua). Cuestionarios de satisfacción}

En el ámbito de la intervención social (Ander-Egg, 2004:7-8), los términos de ayuda mutua se suelen utilizar o bien para referirse a grupos formados de forma voluntaria, donde los miembros comparten necesidades y problemas similares o bien para significar una manera de cooperar para llevar a cabo proyectos o programas comunitarios de acción social.

En nuestro centro, el GAM es un servicio gratuito que se ofrece a los familiares cuidadores de forma quincenal, coordinado por la psicóloga del centro y la trabajadora social. De los objetivos del grupo de ayuda mutua, cabe destacar el soporte psicoemocional a los cuidadores, lo cual les proporciona herramientas para poder afrontar de una forma más sana el cuidado de sus familiares, y de las tareas derivadas del cuidado a la persona enferma. Además, en el GAM pretendemos que las personas que acuden al servicio puedan tener un lugar íntimo y un espacio de contención para compartir sus inquietudes, angustias, preocupaciones, sufrimientos, todo aquello que les supone el tener a su cargo a una persona enferma, y que sientan que no están solos, que hay otras personas en situaciones similares, que comparten y comprenden sus problemas, en definitiva, que sientan que se les apoya. Además, pretendemos que los cuidadores 
afronten la realidad de su situación, que aprendan lo que conlleva la enfermedad, tanto a partir de las experiencias de los otros componentes del grupo como de la implementación del programa psicoeducativo.

En el grupo focal observado se han realizado diversas estrategias y programas de intervención. Al principio se ofreció un programa psicoeducativo basado en las necesidades que los profesionales extrajimos a partir de las entrevistas de ingreso del usuario en el centro. El esquema básico del programa que se realizó durante el periodo 2005-2008 se fue modificando e incluyendo variaciones en el trascurso de las sesiones, en función de los intereses y necesidades observadas en los participantes.

A partir de las necesidades derivadas del grupo y de los resultados que se fueron extrayendo en este periodo de tiempo de la encuesta de satisfacción, valoramos la posibilidad de realizar un tipo de sesiones encaminadas a ofrecer espacios seguros de terapia grupal, combinando sesiones monográficas con profesionales del equipo técnico y alguna sesión de relajación según la técnica de Relajación Progresiva de Jacobson. Esta metodología llevada a cabo en el período 2008-2010, ha obtenido resultados muy beneficiosos para el grupo según han expresado los destinatarios en las encuestas de satisfacción, una vez administrado el cuestionario de satisfacción que cumplimentaron la totalidad de ellos.

El nivel de satisfacción general fue muy bueno y las sesiones fueron valoradas como muy interesantes y útiles. La totalidad del grupo recomendaría estas sesiones en una situación parecida.

Los aspectos más valorados fueron la obtención de información y el reunirse con otras personas y hablar de sus sentimientos; también encontrar apoyo emocional y ser escuchados.

Los cuidadores asistentes al GAM contestaron en su totalidad que sienten que cuidan mejor a sus familiares desde que vienen a estas sesiones. Las respuestas respecto a si creen que se cuidan más ellos también fue favorable en más de la mitad de los casos, el resto indicó que igual que antes.

Los cuidadores creen que están mejor atendidos en el domicilio aunque la respuesta que dieron sobre su opinión en ingresar a su familiar en residencia fue afirmativa; dependerá del grado de deterioro de su familiar. Sin embargo, cuando se les preguntó si ingresaría de inmediato en una residencia la totalidad de la muestra respondió negativamente; eso explica la voluntad de seguir cuidando en el domicilio.

Las fortalezas destacadas del GAM son la posibilidad de establecer relaciones sociales con otras personas en situaciones similares, la comprensión sobre la propia enfermedad, la posibilidad de expresar sentimientos y preocupaciones, el hecho de hacerse cargo de cómo se siente el enfermo y compartir el espacio del grupo para darse cuenta de situaciones similares. 
Como aspectos a mejorar, se destaca la propuesta de un cambio de horario, así como de contenido, por lo que se prevé implementar un programa psicoeducativo. Además se ha observado que las personas que asisten al grupo presentan "pérdidas de memoria" por lo que se propondrá un programa de estimulación cognitiva para los cuidadores, intercalándolo con las sesiones de terapia grupal. Algunos familiares cuidadores - no participantes del grupo- han manifestado no haberles llegado la información sobre el programa, en este sentido pensamos que deberíamos activar los circuitos de difusión.

\section{El cuestionario de sobrecarga del cuidador}

El cuidador/a principal suele padecer síntomas psicológicos, un cierto estrés que conlleva su rol, ansiedad, desánimo, depresión, vergüenza, soledad, tristeza, relación de dependencia, sentimiento de culpa, obligación y aislamiento social. Por este motivo, acostumbramos a administrar los cuestionarios de sobrecarga del cuidador de forma periódica.

En el último año, de los resultados extraídos del test, se resaltó la necesidad de dedicarse más tiempo a sí mismo por una parte de la muestra (aquella que se ha incorporado recientemente al grupo), aunque la mayoría afirma que casi nunca necesitan más tiempo; ahora bien, el 50\% siente que el enfermo reclama más tiempo del que en realidad precisa. Además, la mayoría opina que el enfermo espera casi siempre ser cuidado como si fuera la única persona con la que poder contar, si observamos la gráfica 1. Podría tratarse simplemente de la percepción del cuidador, como única persona capaz de cuidar.

Gráfica 1. Ítem 13. ¿Cree que su familiar espera que Ud. le cuide como si fuera la única persona con la que puede contar?

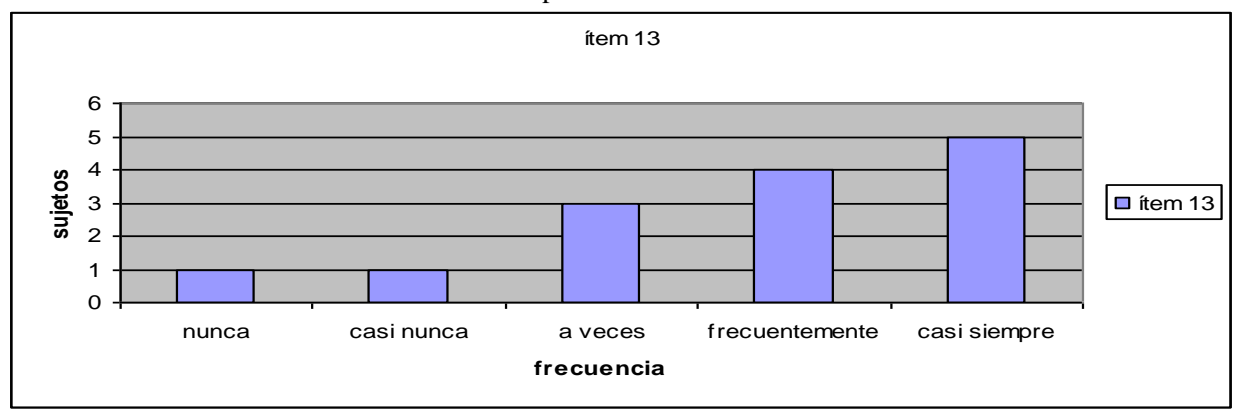

Según los resultados de la siguiente gráfica, sobre si los cuidadores se sienten tensos por tener que cuidar de la persona enferma (ítem 3), observamos que la mayoría opina a veces; lo mismo ocurre delante de la situación de enfado del cuidador (ítem 5), 
aunque los resultados apuntan hacia el extremo nunca. Así pues, se podría afirmar que no existe una alta sobrecarga del cuidador, si observamos éstos dos ítems.

Gráfica 2. Comparativa de ítems: tensión del cuidador y enfado

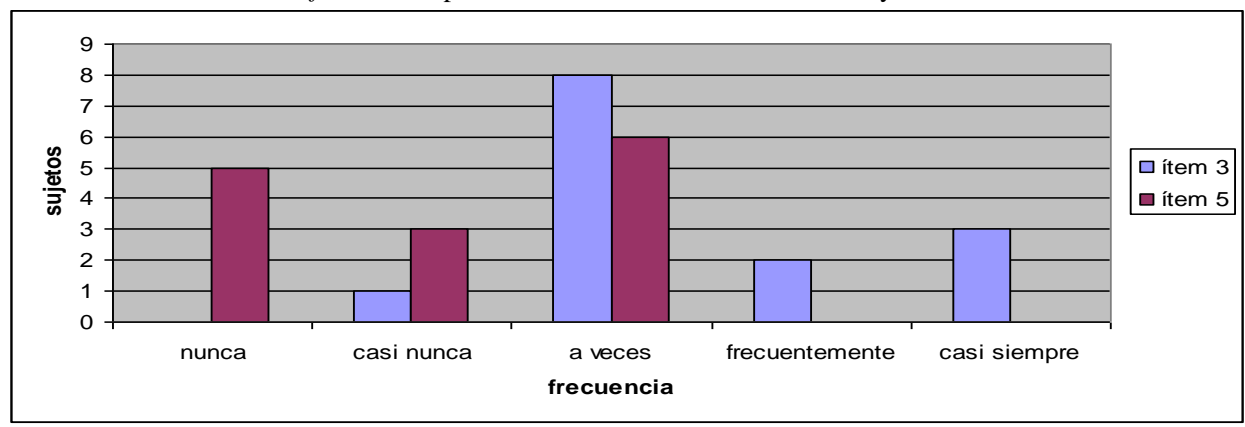

Ante la sensación de agobio experimentada por el cuidador (ítem 9), parece ser que la muestra se sitúa entre casi nunca y a veces; en cambio, responden que con frecuencia creen que su salud se ha resentido al cuidar de su familiar (ítem 10).

Gráfica 3. Comparativa de ítems: estar agobiado y sentir que la salud está resentida

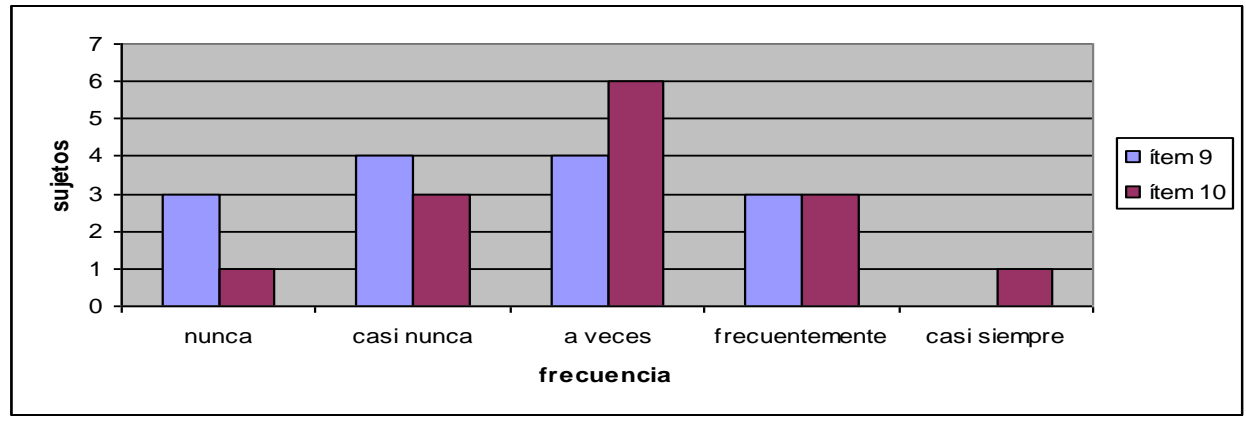

De las reflexiones derivadas de los datos analizados, sí se observa que después de haber asistido de forma continuada al grupo de ayuda mutua, los cuidadores manifiestan un grado menos elevado de estrés. Al mismo tiempo parece que apuestan más por sus propios cuidados, valorando -algunos asistentes- la necesidad de crearse espacios propios. Ahora bien, muchos de los cuidadores sienten que sin ellos sus personas queridas no estarían tan bien cuidadas, a pesar de resentirse su salud física, o de sentirse agobiados. 


\section{CONCLUSIONES}

Del análisis de los datos, se puede observar la necesidad de realizar desde las instituciones acciones para prestar atención al cuidador del enfermo de Alzheimer, a partir de diversas estrategias. En este artículo hemos querido mostrar aquellas acciones que realizamos en nuestro Centro y que nos han ofrecido buenos resultados en todo este tiempo. A partir de dichas acciones y la de la formación psicoeducativa, hemos destacado cómo la permanencia del usuario en el domicilio es de mejor calidad y se puede llegar a prolongar en el tiempo.

Además, a partir de los datos extraídos parece sugerirse que las acciones dirigidas a los cuidadores mejoran sus propias acciones, su manera de hacer, su práctica con la persona enferma, mejorando la calidad de atención del propio cuidador y en consecuencia la de la persona que está cuidando. En todo caso, la decisión de ingresar en un centro residencial, es tomada de forma reflexiva y más distanciada, sin tantas emociones negativas.

Creemos pues que es necesario que los políticos se conciencien con las necesidades de personas con enfermedad de Alzheimer y las de sus cuidadores, y que ofrezcan políticas respetuosas con las necesidades de la sociedad. Para ello, los centros y servicios residenciales y centros de día, debieran ofrecer al personal espacios, tiempos y presupuesto para dedicarse a dichas investigaciones, valorando éstas acciones para mejorar la calidad de los servicios.

La intervención interdisciplinaria es el eje a partir del cual los programas encaminados a los familiares, cuidadores, y enfermos, pueden tener éxito. Hemos presentado una experiencia de trabajo en equipo, el trabajo interdisciplinar de la Residencia y Centro de Día de Horta como ejemplo de acción a través del cual se mejora la calidad de actuación profesional, por lo tanto la calidad de vida del cuidador y del usuario y en consecuencia la calidad del centro. Es pues imprescindible la implicación de los profesionales en un trabajo de ésta envergadura, y para ello es necesario que los directivos de los centros residenciales y centros de día, inicien ésta estrategia de trabajo como punto de partida para la acción.

Además, creemos que trabajar de forma interdisciplinaria supone mejorar las acciones que se proponen en los centros, una optimización de iniciativas previas dirigidas a reducir el malestar a los cuidadores (Losada y otros, 2006, 2008; González y otros, 2007). En este sentido, nuestra experiencia muestra la necesidad de seguir interviniendo desde las disciplinas que hemos expuesto, la psicología, el trabajo social, la terapia ocupacional, la fisioterapia y la enfermería.

A partir de los resultados extraídos de la muestra del grupo focal, pensamos que el GAM proporciona precisamente aquella red de relaciones sociales que las personas cuidadoras ha ido perdiendo a lo largo del tiempo, estimulando su posibilidad 
de crear nuevas relaciones con personas que comparten muchos aspectos comunes en su vida diaria. Pero además el grupo ayuda a traspasarse informaciones útiles sobre temas derivados del cuidado del enfermo y del autocuidado del cuidador, lo cual permite crear transferencias informativas basadas en la confianza.

En cuanto a las posibles vías de intervención, nos planteamos crear un programa de estimulación cognitiva enfocado en los cuidadores de personas enfermas, después de las necesidades recogidas por los beneficiarios del GAM, realizando la suficiente difusión. Así como volver a implementar un programa psicoeducativo para dar respuesta a las demandas principales de los cuidadores incluyendo la perspectiva de los diferentes profesionales y áreas a tratar de modo que se les pueda ofrecer una información des de la interdisciplinariedad.

En cuanto a las limitaciones de los beneficios que hemos mostrado en este estudio, está implicada la reflexión que hemos ido realizando a lo largo del trabajo, y que se refiere al tiempo limitado de atención al cuidador, pues en nuestro caso la atención se dispensa mientras la persona dependiente es usuaria del Centro. Una vez se decide que el enfermo se traslada a otra residencia, o fallece, el cuidador, progresivamente, va abandonando progresivamente el recurso que ofrecemos.

En definitiva, pensamos que atender y ofrecer herramientas a los cuidadores de personas enfermas es una parte importante en nuestro trabajo, desde las diferentes disciplinas, puesto que mejora la calidad de vida del propio cuidador, y en consecuencia la de la persona enferma, optimizando su estancia en el domicilio, y además mejora la calidad del servicio que ofrece estos recursos. Faltaría pues una implicación de las administraciones para satisfacer las demandas de los usuarios y para ofrecer recursos a este tipo de estrategias.

\section{REFERENCIAS}

Ander-Egg, E. (2004). Los grupos de auto ayuda y el apoyo social. Barcelona: Ergon.

Artaso, B., Goñi, A. y Gómez, A.R. (2001). Sobrecarga del cuidador informal del paciente con demencia: demanda en un Centro de Día Psicogeriátrico en Navarra. Geriátrika, 17, 3943.

Córdoba, R. (2010 ). La brújula del cuidador. Ayuda para familiares y cuidadores. Barcelona: Plataforma actual.

Crespo, M. y Martínez, J.L. (2007). El estrés en cuidadores de mayores dependientes. Madrid: Pirámide.

Diputació de Barcelona (2004). El suport a les persones cuidadores: una estratègia de conciliación. Barcelona: Institut d'edicions de la Diputació.

Leturia, F.J. y Yanguas, J.J. (1999). Las personas mayores dependientes y la complementación sociosanitaria: un reto de futuro. En F.J. Leturia, J.J. Yanguas y M. Leturia (Coords.), Las personas mayores y el reto de la dependencia en el siglo XXI (pp. 67-83). San Sebastián: Departamento de Servicios Sociales de la Diputación Social de Guipúzcoa. 
Losada, A., Montorio, I., Izal, M. y Márquez-González, M. (2006). Estudio e intervención sobre el malestar psicológico de los cuidadores de personas con demencia. El papel de los pensamientos disfuncionales. Madrid: IMSERSO.

Losada, A., Peñacoba, C., Márquez-González, M. y Cigarán, M. (2008). Cuidar cuidándose. Evaluación e intervención interdisciplinar con cuidadores familiares de personas con demencia. Madrid: Encuentro.

Mace, N. y Rabins, P. (2004). El día de 36 horas. Una guía práctica para las familias y cuidadores de enfermos de Alzheimer, otras demencias seniles y pérdida de memoria. Madrid: Paidós.

Márquez-González, M., Losada, A., Izal, M., Pérez-Rojo, G. y Montorio, I. (2007). Modification of Dysfunctional Thoughts about Caregiving in Dementia Family Caregivers: description and outcomes o fan Intervention Program. Aging \& Mental Heath, 11, 616625.

Pérez, J.M. (1997). Evolución vivencial del cuidador. Psiquis, 18, 138-141.

Tejedor, O. (2011). Cuidadores. Documental: España.

Trallero, C. y Oller, J. (2008). Cuidados musicales para cuidadores. Bilbao: Desclée de Brouwer.

Recibido: 1 de julio de 2011 Recepción Modificaciones: 1 de septiembre de 2011 Aceptado: 2 de septiembre de 2011 